\title{
Balancing elliptic curve coprocessors from bottom to top
}

\author{
Niels Pirotte ${ }^{1}$, Jo Vliegen ${ }^{1}$, Lejla Batina ${ }^{2}$ and Nele Mentens ${ }^{1}$ \\ ${ }^{1}$ ESES and imec-COSIC/ESAT, KU Leuven, Belgium, \\ firstname. lastname@kuleuven.be \\ ${ }^{2}$ Digital Security Group, Radboud University, the Netherlands, \\ lejla@cs.ru.nl
}

\begin{abstract}
In 2016, Renes et al. were the first to propose complete addition formulas for Elliptic Curve Cryptography (ECC) on Weierstrass curves. With these formulas, the same set of equations can be used for point addition and point doubling, which makes software and hardware implementations less vulnerable to sidechannel (SCA) attacks. Further, all inputs are valid, so there is no need for conditional statements handling special cases such as the point at infinity.

This paper presents the first ASIC design of the complete addition formulas of Renes et al. Each computation layer in the design is balanced, from the field arithmetic to the point multiplication. The design explores two datapaths: a full-width Montgomery Multiplier ALU (MMALU) with a built-in adder and a serialized version of the MMALU. The interface sizes of the MMALU are optimized through an exploration of the design parameters. The register file size is minimized through an optimal scheduling of the modular operations. The top-level point multiplication is implemented using the Montgomery ladder algorithm, with the additional option of randomizing the execution order of the point operations as a countermeasure against SCA attacks. The implementation results after synthesis are generated using the open source NANGATE45 library. Keywords: ASIC, Weierstrass curve, complete addition formulas, elliptic curve cryptography (ECC), side-channel analysis (SCA), simple power analysis (SPA), differential power analysis (DPA), Montgomery ladder
\end{abstract}




\section{Introduction}

The rapid growth of the internet of things (IoT) introduces two main security challenges. Firstly, securing the communication between two or more entities requires cryptographic algorithms and protocols to be implemented on 5 IoT devices. This raises the challenge of adding cryptography to hardware and/or software devices that are constrained in computational resources and power/energy consumption. Secondly, IoT devices are often physically accessible to potential malicious users, making them vulnerable to side-channel analysis attacks (SCA) 11. Examples of side-channels are the power consumption, the timing behavior and the electromagnetic radiation of the device. SCA attacks exploit leakage through these channels to extract secret information originating from the physical implementation of cryptographic algorithms and protocols.

In this work, we consider both resource-constrained and side-channel protected implementations of Elliptic Curve Cryptography (ECC) 2] 3]. This type of public-key cryptography is preferred in constrained devices, thanks to smaller key sizes than e.g. RSA [4], leading to a reduction of the power consumption and the computational resources. ECC was introduced in 1985 and 1987 independently by Victor Miller [2] and Neal Koblitz [3], respectively. They proposed special discrete-log based cryptosystems using a group of points on an elliptic curve (EC) (plus a special point, the so-called point at infinity) as the underlying group with a corresponding point addition law. In general, this addition law requires different equations for a pair of identical points and for a pair of different points. These equations are called point doubling and point addition, respectively. The most straightforward way of implementing an EC point multiplication, i.e. the basic operation in an EC-based cryptosystem, is through iterative conditional branching of point doublings and point additions. This is disadvantageous for SCA resistance, because the conditional branching reveals information on the executed operation. This weakness was exploited by Örs et al. [5] on an FPGA implementation of ECC in a dedicated power analysis setup; they managed to retrieve the secret key through Simple Power Analysis (SPA). 
In [6], Bernstein and Lange derive complete addition formulas, which use the same set of equations for point doubling and point addition. This leads to constant-time and exception-free implementations, mitigating the behavioral effects of branching. While the authors of [6] present their formulas for specific types of curves, namely Edwards curves, Renes et al. [7] are the first to propose complete addition formulas for the broadly used Weierstrass curves over prime fields. They also notice that in some cases, performance loss will be acceptable in comparison to traditional incomplete formulas.

Our contributions. This work is the first to present an ASIC design of bottom (i.e., the datapath) to top (i.e., the control logic for the point multiplication) to protect the design against timing and SPA attacks. This results in an inherently balanced implementation (without dummy operations) of ECC over Weierstrass curves. The following design choices and optimizations are im-

45 plemented: (1) two versions of the datapath, namely a full-word Montgomery Multiplier ALU (MMALU) with integrated adder functionality and a serialized version of the MMALU; (2) an optimal input and output size of the MMALU through a careful exploration of the design parameters and bounds, which also has an influence on the operation count in the point addition formulas; (3) a 50 register file with minimal depth, by reducing the number of writable registers (through the intelligent use of shift registers), and by optimizing the size of the input multiplexers (by limiting left-operand and right-operand accessibility); (4) a second version of the ECC implementation with randomized point operations in the Montgomery ladder, enhancing the protection against Differential Power ${ }_{55}$ Analysis (DPA) attacks.

Our work extends the balanced ECC coprocessor of Pirotte et al. in [8]. It gives a more detailed explanation of the design process, more particularly on the lifetime analysis of the intermediate values and their mapping onto the register file. Another extension beyond the work presented in [8] is the design

${ }_{60}$ of an area-reduced version of the coprocessor through an optimization of the datapath. 
Organization. Section 2 gives a brief overview of the necessary preliminaries and used notations. In Sect. 3, a summary of related literature on complete formula implementations is given. Next, Sect. 4 discusses the design choices and the experimental setup. Section 5 elaborates on the implementation results and discusses the side-channel resistance of the coprocessor. Finally, Sect. 6 concludes the paper and identifies some avenues for future research.

\section{Preliminaries}

ECC is based on a group structure on an EC. An EC is a set of points $(x, y)$, which are solutions of a polynomial equation defined over a Galois field of the following form:

$$
y^{2}=x^{3}+a x+b
$$

This equation holds for so-called short Weierstrass curves, which are isomorphic to every possible elliptic curve over the same field. Prime fields, i.e. $G F(p)$, and binary extension fields, i.e. $G F\left(2^{n}\right)$, are the most commonly used finite fields in the context of ECC. In this paper, only prime fields are considered and therefore $a$ and $b$ in Eq. (1) are constants in $G F(p)$ and $p$ is a large prime.

Next, an additive Abelian group can be obtained by defining an addition law $(+)$ on an EC that is nonsingular, requiring $4 a^{3}+27 b^{2} \neq 0 \bmod p$. The elements in the group are the points on the EC, along with an additional point called the point at infinity. The point at infinity is denoted by $\mathcal{O}$ and can be expressed as $\mathcal{O}=(x, \infty)$. The obtained Abelian group can be represented as follows:

$$
(\{(x, y) \mid x, y \in G F(p) \text { satisfying Eq. (1) }\} \cup \mathcal{O},+)
$$

The addition law can be implemented using the following operations modulo $p$ : addition, subtraction, multiplication and inversion. In hardware, inversions modulo $p$ are very costly operations. Nevertheless, they can be avoided when embedding the elliptic curve in the projective plane, i.e. $\mathbb{P}^{2}(G F(p))$. In order to do so, every point $(x, y)$ is mapped to $(x: y: 1)$ and the point at infinity is mapped to $(0: 1: 0)$. The projections $(x: y: z)$ and $(\lambda x: \lambda y: \lambda z)$ are 
equal, and thus $\left(\frac{x}{z}: \frac{y}{z}: 1\right)$ equals $(x: y: z)$. When embedding the EC in the projective space, Eq. (1) becomes

$$
y^{2} z=x^{3}+a x z^{2}+b z^{3} .
$$

A series of point additions is called a point multiplication or scalar multiplication and is defined as follows:

$$
m P=\underbrace{P+P \cdots+P}_{m \text { times }}
$$

with $P$ an element of an EC group structure and $m$ a positive integer. For negative $m$, Eq. (4) becomes $m(-P)$. Point multiplication is used in ECC schemes, because the security relies on the elliptic curve discrete logarithm problem (ECDLP). The ECDLP states that it is unfeasible to calculate $m$, such that $Q=m P$, when the points $P$ and $Q$ are known.

A commonly used technique to design implementations of such ECC schemes,

80 is a bottom-up design approach, wherein each abstraction level uses the operations of the abstraction level below. Figure 1 depicts the different abstraction levels for ECC applications. The remainder of this section discusses the different abstraction levels up to the point multiplication and introduces the algorithms we use in our design.

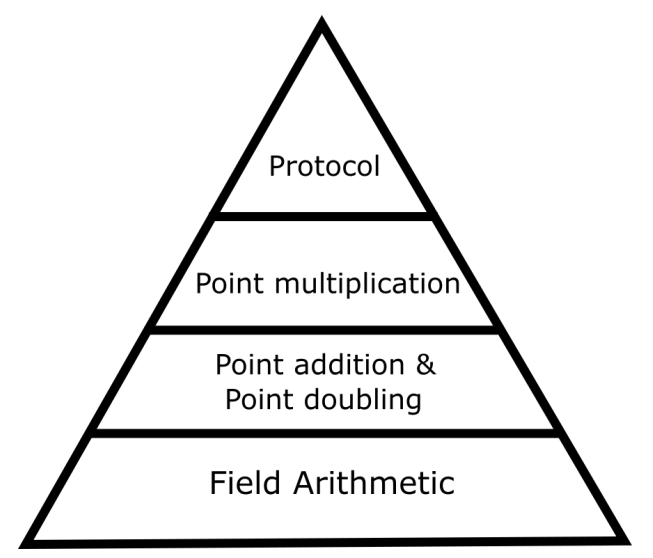

Figure 1: Abstraction levels in the design of an ECC coprocessor. 


\subsection{Field Arithmetic}

At the lowest level are the field arithmetic operations. When using the projective representation of the EC, only modular addition, modular subtraction and modular multiplication suffice to implement the addition laws. Modular multiplication modulo $p$ is significantly more complex than modular addition and subtraction, due to the required trial divisions. Therefore, a variety of techniques were designed to speed up or scale down this operation in hardware. For modular multiplication, Montgomery multiplication is a popular technique with a small chip area, a low power consumption and a high throughput in mind. The Montgomery Modular Multiplication (MMM) algorithm was introduced by Peter Montgomery in 1985 [9]. In 1999, Colin Walter suggested an improvement, which made the final reduction at the end of the original algorithm unnecessary by introducing modified input bounds as well as a lower bound for the Montgomery parameter [10]. This improvement is advantageous for SCA resistance since it leads to a time-constant implementation. The Montgomery multiplication algorithm, which we use in our design, is given in Alg. 1 .

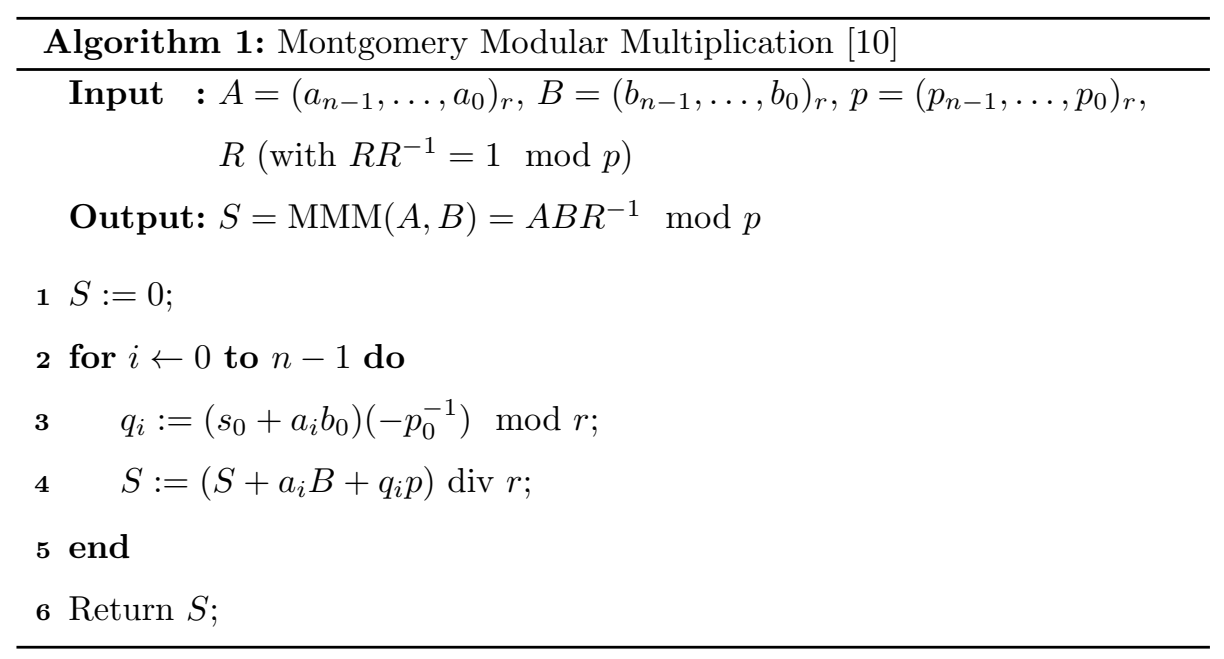

In Alg. 1, $a_{i}$ stands for the $\mathrm{i}$-th digit of the word $A$. When $A$ is an n-digit number expressed in base $r, A$ can be written as $A=\sum_{i=0}^{n-1} a_{i} r^{i} . B$ and $p$ are represented in a similar way. The power of the Montgomery algorithm is the 
ability to calculate a modular operation by replacing costly trial divisions by a

prime with divisions by a power of 2 . The latter comes for free in hardware, by implementing logical shift operations. MMM, working on the input operands $A$ and $B$, computes $A B R^{-1} \bmod p$, where $p$ is the modulus and $R^{-1}$ is an element of the finite field satisfying $R R^{-1}-p p^{\prime}=1$ or identically $R R^{-1}=1$ $\bmod p . R$ is a power of two, i.e. $r^{n}$, such that $R=r^{n}>p ; R$ is also called the

Montgomery parameter. The algorithm becomes useful when the operands are first transformed to Montgomery representation, mapping $A$ and $B$ to respectively $A_{\text {Mont }}=A R \bmod p$ and $B_{\text {Mont }}=B R \bmod p$. This way, Montgomery multiplication can be used to calculate modular multiplications, because the algorithm ensures $S_{\text {Mont }}=S R \bmod p=\operatorname{MMM}\left(A_{M o n t}, B_{\text {Mont }}\right)$, with $S=A B$ $115 \bmod p$. At the end of the calculations in the Montgomery domain, the result needs to be converted back. As a consequence, Montgomery multiplication requires two additional steps. However, this overhead becomes negligible when a large series of consecutive operations is performed, which is the case in the context of scalar multiplication 1 p

\subsection{Point Addition \& Point Doubling}

The next abstraction layer involves the point operations, namely point addition and point doubling. As mentioned in Sect. 1, point doubling and point addition use a different set of equations in most addition laws. When implemented in hardware or software, this leads to different execution times and power traces for both, inevitably leaking information on the scalar $m$ in the computation of the point multiplication $m P$. Evidently, this side-channel leakage needs to be avoided to avert weakening the implemented ECC scheme.

Therefore, we use the complete addition law, as proposed in [7], which uses the same addition formulas for point addition and point doubling. More specif30 ically, we use Algorithm 7 in [7], since it has a minimal number of operations

\footnotetext{
${ }^{1}$ Bézout's identity ensures the existence of $R^{-1} 9$ [0.

${ }^{2} R^{-1}$ and $p^{\prime}$ can be calculated using the extended Euclidean algorithm.
} 
and no input restrictions. This algorithm targets short Weierstrass curves with $a=0$, i.e. $j$-invariant 0 curves. These curves are also used in practice, such as the secp256k1 curve used in the Bitcoin Protocol [11.

The considered formulas of Algorithm 7 in [7, giving new point coordinates $\left(X_{3}: Y_{3}: Z_{3}\right)$ dependent on input points $\left(X_{1}: Y_{1}: Z_{1}\right)$ and $\left(X_{2}: Y_{2}: Z_{2}\right)$, are

$$
\begin{aligned}
X_{3}= & \left(X_{1} Y_{2}+X_{2} Y_{1}\right)\left(Y_{1} Y_{2}-3 b Z_{1} Z_{2}\right) \\
& -3 b\left(Y_{1} Z_{2}+Y_{2} Z_{1}\right)\left(X_{1} Z_{2}+X_{2} Z_{1}\right), \\
Y_{3}= & \left(Y_{1} Y_{2}+3 b Z_{1} Z_{2}\right)\left(Y_{1} Y_{2}-3 b Z_{1} Z_{2}\right) \\
& +9 b X_{1} X_{2}\left(X_{1} Z_{2}+X_{2} Z_{1}\right) \\
Z_{3}= & \left(Y_{1} Z_{2}+Y_{2} Z_{1}\right)\left(Y_{1} Y_{2}+3 b Z_{1} Z_{2}\right) \\
& +3 X_{1} X_{2}\left(X_{1} Y_{2}+X_{2} Y_{1}\right)
\end{aligned}
$$

\subsection{Point Multiplication}

The most straightforward algorithm for point multiplication is the doubleand-add algorithm, consisting of consecutive point additions and point doublings. To overcome the side-channel leakage caused by the conditional branches for point multiplication. The Montgomery ladder, as presented by Peter Montgomery in [12, is shown in Alg. 2. In contrast with the double-and-add method, each iteration executes the same operations, namely one point addition and one point doubling.

145 then a point doubling is performed, independent of $m_{i}$. In 13, the authors introduce additional randomization by using a random bit which decides on the execution order of both operations. This order introduces an additional uncertainty independent of the key, further complicating SCA attacks. The resulting Montgomery ladder is shown in Alg. 3 and is applied in our ASIC design. 


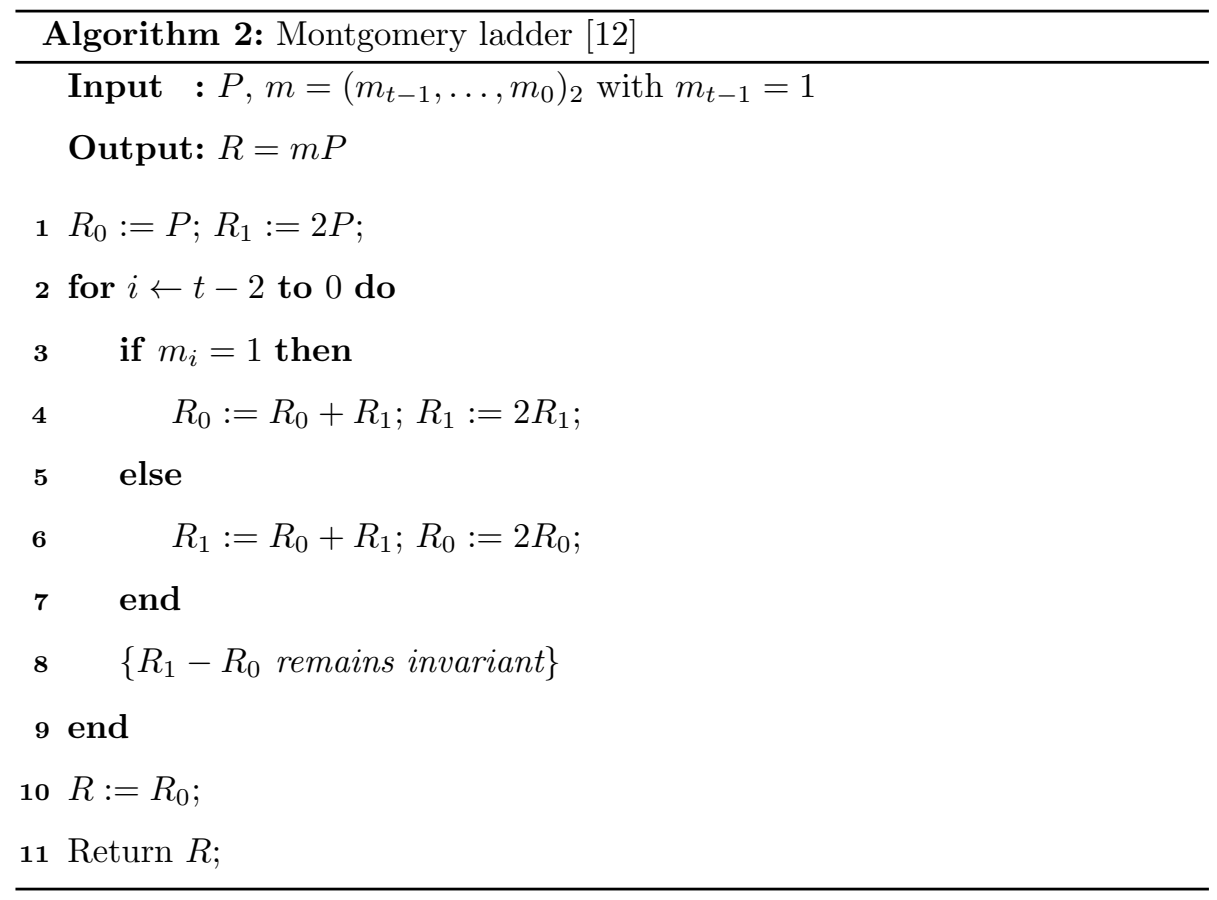

\section{Related Work}

The first work to introduce a fully balanced ECC implementation was by Batina et al. [14. The authors modified the non-complete addition formulas over binary extension fields in order to make them balanced. For point multiplication, the Montgomery ladder algorithm was used. The architecture was implemented on an FPGA and the resistance against an SPA attack was evaluated. A similar method was applied to Edwards curves in [13.

Examples of elliptic curves with complete addition laws are Edwards curves [6], twisted Edwards curves [15] and twisted Hessian curves [16. The work of Renes et al. 7] was the first to propose complete addition formulas on standardized i.e. Weierstrass curves over prime fields.

In [17, Massolino et al. present the first FPGA implementation of the formulas in [7. The result is a competitive design emphasizing parallelization possibilities. The design executes a number of field operations simultaneously by using two up to six processors, which increases throughput, but also silicon 


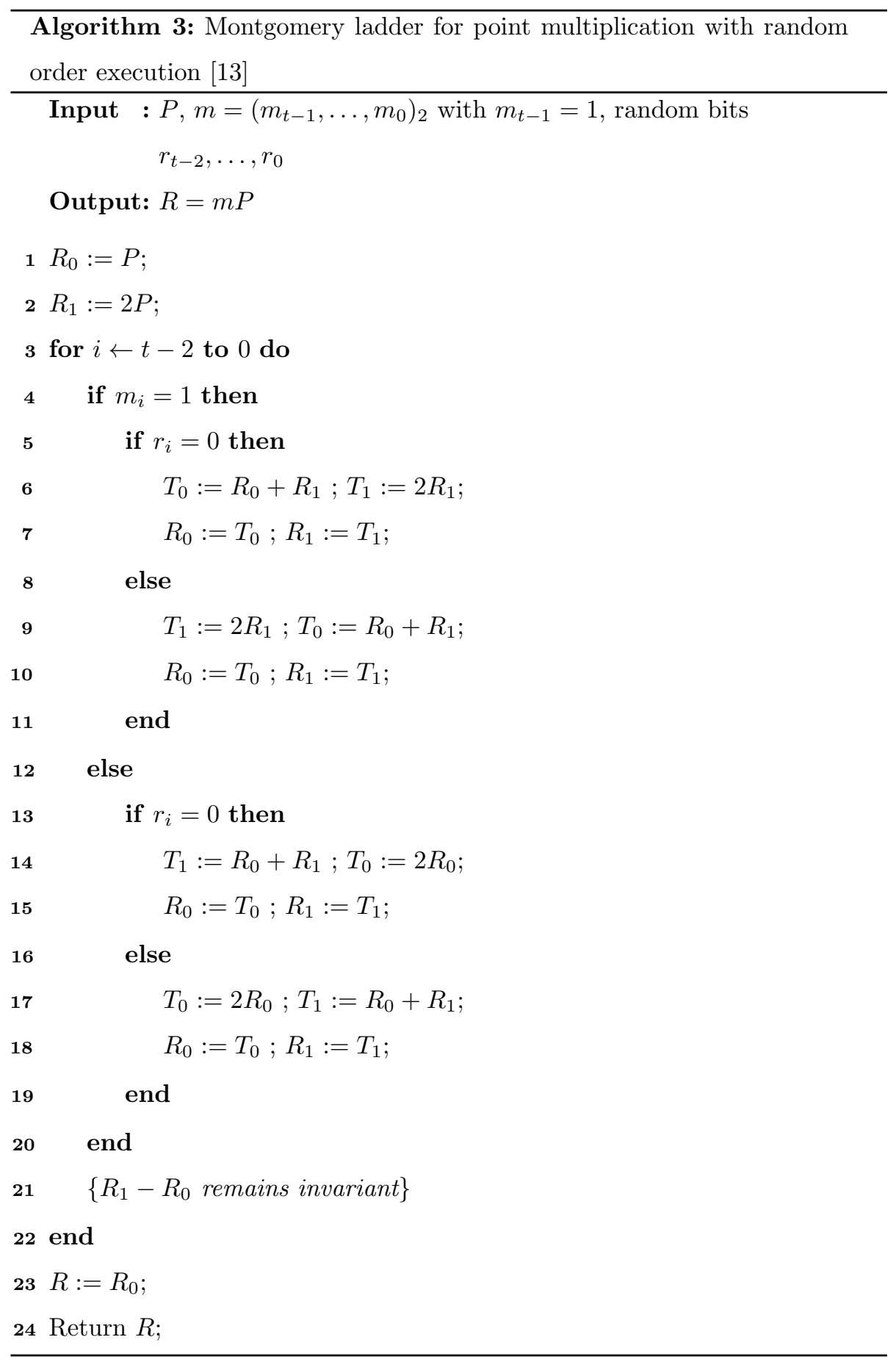


area. In [18], Chmielewski et al. implement and evaluate three FPGA implementations of the formulas in [7]: a non-protected architecture and two architectures protected by randomization countermeasures for DPA protection.

Our work realizes the first ASIC implementation of the complete formulas of Renes et al. [7]. The paper extends the work of Pirotte et al. [8] by giving more details on the design process and providing a second architecture with an area-optimized datapath.

Related work that implements different types of curves, namely binary Edwards curves, but also concentrates on the optimization of the internal point addition and doubling operations and performs register lifetime analysis, is presented in [19].

\section{Design}

This section elaborates on the functionality and design choices of the ASIC implementation in a bottom-up way, following the abstraction layers in Fig. 1 . After giving an overview of the design parameters of full-word Montgomery multipliers, the Montgomery Modular ALU (MMALU) is discussed, which enables modular multiplication, addition and subtraction. Hereafter, the design choices for the control logic, implementing the complete point addition formulas

185 in Eq. (5), are explained. Finally, the Montgomery ladder implementation for point multiplication is discussed.

\subsection{Design Parameters of the $M M M$}

This paragraph discusses the influence of certain design parameters on the function of Alg. 1] inspired by the doctoral thesis of Lejla Batina [20. Especially important is the relation between the input bounds and the Montgomery parameter $R$. This relationship influences speed and area, because it is directly linked to the required number of iterations of the algorithm and the size of the input and output registers. To understand the influence of these parameters, the operation of the algorithm is explained below. For simplicity, it is assumed that all data are expressed in binary form and therefore the base $r$ is 2 . 


\subsubsection{Working principle}

The correctness of the MMM algorithm depends on two statements, both easily verified via induction. The first statement,

$$
0 \leq S<p+B
$$

holds during each operation of the for loop in Alg. 1 and ensures the output remains bounded. After $n$ iterations, $S=A B R^{-1} \bmod p$ with $R=r^{n}$. This is verifiable with the help of the following statement, in which $Q=\left(q_{n-1}, \ldots, q_{0}\right)$ (cfr. Alg. 1):

$$
R S=A B+Q p \Rightarrow S=A B R^{-1} \bmod p
$$

\subsubsection{Divisibility by 2}

In Alg. 1, addition with $q_{i} p$ always ensures an outcome divisible by 2 in the last step of the for loop. In other words, the least significant bit (LSB) of the outcome on line 4 is 0 . This is easily validated by replacing $q_{i}$ with the expression on line 3 in Alg. 1. As a result, the LSB of $S$ becomes:

$$
\begin{aligned}
& s_{0}+a_{i} b_{0}+q_{i} p_{0} \\
= & s_{0}+a_{i} b_{0}+\left(\left(s_{0}+a_{i} b_{0}\right)\left(-p_{0}^{-1}\right)\right) p_{0} \\
= & s_{0}+a_{i} b_{0}+\left(s_{0}+a_{i} b_{0}\right)(-1) \\
= & 0
\end{aligned}
$$

\subsubsection{Upper Bound on $Q$}

$Q$ cannot be bigger than $R-1$, because $Q$ has maximum $n$ bits and therefore has a maximum value of $r^{n}-1=R-1$.

\subsubsection{Upper Bound on the Inputs}

A design parameter with significant influence on the implementation is the bound on the inputs; it directly determines the size of the inputs, the output and the intermediate registers in the implementation. Let us assume that the inputs are bounded by a multiple $k$ of the modulus $p$ :

$$
A, B<k p \stackrel{\mathrm{Eq} \cdot[6}{\Rightarrow} S<p+k p=(k+1) p
$$


Thus, when the inputs are smaller than $k$ times the modulus $p$, the input registers need to store $\left\lceil\log _{r}(k)\right\rceil$ more bits than the number of bits needed to represent $p$. Further, the intermediate result needs $\left\lceil\log _{r}(k+1)\right\rceil$ bits more than $p$.

\subsubsection{Lower Bound on the Montgomery Parameter}

The Montgomery parameter $\left(R=r^{n}\right)$ determines the number of iterations $(n)$ to obtain the result. We assume that the lower bound on the Montgomery parameter is a multiple $l$ of $p$.

\subsubsection{Upper Bound on the Output}

The relation between the Montgomery parameter and the input bound has an important effect on the output of the MMM. Assuming that both the inputs and the Montgomery parameter are bounded by a multiple $(<k p$ and $>l p$, respectively) of $p$, we obtain the following bound on $S$ :

$$
\begin{aligned}
& A, B<k p \text { and } R>l p \\
\stackrel{\text { Eq. } 7}{\Rightarrow} & S=\frac{A B}{R}+\frac{Q p}{R} \\
\Rightarrow & S<\frac{k^{2} p^{2}}{R}+\frac{(R-1) p}{R} \\
\Rightarrow & S<\frac{k^{2} p^{2}}{l p}+\frac{(R-1) p}{R} \\
\Rightarrow & S<\frac{k^{2}}{l} p+p-\frac{p}{R} \\
\Rightarrow & S<\left(\frac{k^{2}}{l}+1\right) p
\end{aligned}
$$

As an example, assume that the input bounds are $A, B<2 p$ and the Montgomery parameter is larger than $4 p$. Then the output remains smaller than $2 p$, in accordance with the original result in the paper of Walter, enabling MMM without final subtraction [10].

\subsubsection{Summary}

Table 1 gives an overview of the design parameters of the MMM for a prime of $x$ bits. By carefully selecting the lower bound of the Montgomery parameter $(l p)$ and the upper bound of the inputs $(k p)$, the number of iterations $(n)$ and 


\begin{tabular}{|l|c|l|}
\hline Parameter & \multicolumn{1}{|c|}{ Bound } & \# bits \\
\hline Prime $(p)$ & - & $\boldsymbol{x}$ \\
\hline Montgomery parameter $\left(R=r^{n}\right)$ & $>\boldsymbol{l} \boldsymbol{p}$ & $n+1$ \\
\hline Inputs & $<\boldsymbol{k p}$ & $x+\left\lceil\log _{2}(k)\right\rceil$ \\
\hline \# iterations & $=n$ & $\left\lceil\log _{2}(n)\right\rceil$ \\
\hline Intermediate result (after shift) & $<(k+1) p$ & $x+\left\lceil\log _{2}(k+1)\right\rceil$ \\
\hline Intermediate result (before shift) & $<2(k+1) p$ & $x+\left\lceil\log _{2}(k+1)\right\rceil+1$ \\
\hline Output & $<\left(\frac{k^{2}}{l}+1\right) p$ & $x+\left\lceil\log _{2}\left(\frac{k^{2}}{l}+1\right)\right\rceil$ \\
\hline
\end{tabular}

Table 1: Influence of the boundaries of the MMM design parameters on register sizes

the bound on the output and the intermediate values can be determined. These bounds determine the register sizes in the design and are therefore important design parameters.

\subsection{Montgomery Modular ALU}

Most literature on Montgomery multipliers in hardware is focused on fast implementations of the MMM algorithm, often at the expense of area efficiency. For these fast implementations, systolic arrays (e.g. 21, 22]), pipelining (e.g. 23]), high-radix approaches (e.g. 24]) and the use of a Carry-Save Adder (SCA) 25] are very popular. In this paper, we implement two different versions of the Montgomery multiplication algorithm without final subtraction [10]. The first design is inspired by the Modular ALU (MALU) design of Sakiyama et al. 26] with a datapath width equal to the full word length of the operands. The second design is a serialized version that features a reduced area footprint. We discuss the design of both implementations.

\subsubsection{Full-word Design of the MMALU}

As discussed in Sect. 2.1, the MMM has a bounded output dependent on the upper bound of the inputs and the lower bound of the Montgomery parameter $R$. When the inputs of the MMM are limited to numbers smaller than $4 p$, 


\begin{tabular}{|l|l|l|}
\hline & Upper limit & Number of bits \\
\hline Prime & $p$ & $k$ \\
\hline Montgomery parameter & $2^{(k+4)}(>16 p)$ & $k+5$ \\
\hline Inputs & $4 p$ & $k+2$ \\
\hline$\#$ iterations & $k+4$ & $\left\lceil\log _{2}(k+4)\right\rceil$ \\
\hline Intermediate result (after shift) & $5 p$ & $k+3$ \\
\hline Intermediate result (before shift) & $10 p$ & $k+4$ \\
\hline Output & $2 p$ & $k+1$ \\
\hline
\end{tabular}

Table 2: Necessary recourses of the MMALU, with corresponding upper bounds for $R>16 p$

the intermediate results of Alg. 1 are numbers smaller than $5 p$ (after the shift operation). If we choose the Montgomery parameter to be larger than $16 p$, the output is bounded by $2 p$. Table 2 summarizes the previous reasoning.

The functionality of the MMALU can be selected using two control signals, cmd and sub. This can also be seen in Fig. 2, When $c m d$ is set to 0, a MMM is performed on the input operands. When operating in MMM mode, the sub control signal selects either the MMM of the two input operands, i.e. when $s u b$ equals 0 , or otherwise the MMM of one input operand with the second input operand equal to 1 . The second feature can be used to scale down the input operand to ensure an outcome smaller than or equal to $p$, because

$$
\begin{array}{rl} 
& A<4 p \text { and } B=1 \text { and } R>16 p \\
\stackrel{\text { Eq. }}{\Rightarrow} 7 & R S=A B+Q p \\
\Rightarrow & R S<4 p \cdot 1+(R-1) p \\
\Rightarrow & R S<(R+3) p \\
\Rightarrow & S<p+\frac{3 p}{16 p} \\
\Rightarrow & S \leq p
\end{array}
$$

Normally, the MMM of an input operand and 1 transforms the input from the Montgomery representation back to the original domain. However, when 
performed on all coordinates of a point on the EC, this operation only results in a scaling. Remember that the complete formulas to be implemented have inputs and an output in the projective space. Consequently, the formulas yield the same output for scalar multiples of either of the input points, i.e. the output remains unaffected when loading a set of inputs $(X, Y, Z)$ or $(\lambda X, \lambda Y, \lambda Z)$ for either input point. It should also be noted that this property renders transformation of the input coordinates, i.e. $\left\{X_{1}, Y_{1}, Z_{1}, X_{2}, Y_{2}, Z_{2}\right\}$, to their Montgomery representation and transformation of the output coordinates back to the original domain unnecessary. This follows directly from $(X: Y: Z)=(R X: R Y: R Z)$.

Fig. 3 gives a conceptual view of the MMALU when $c m d$ equals 0 . This is a straightforward implementation of Alg. 1 with two ripple carry adders (RCA).

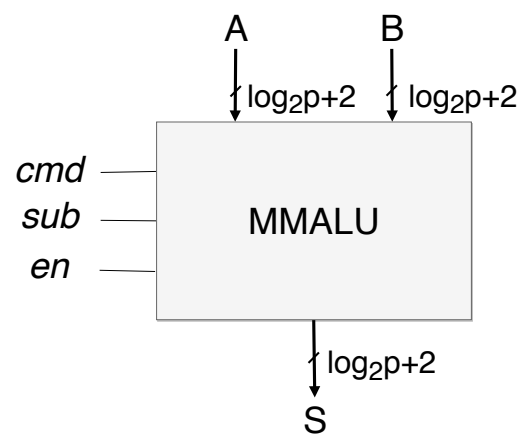

Figure 2: Interface of the MMALU functional block.

Figure 4 shows the functionality of the MMALU when $\mathrm{cmd}=1$. In this mode, the integrated adder functionality is selected. As a result, two field element inputs can be added or subtracted depending on the value of $s u b$. When $s u b$ equals 0 , the adder adds two $2 p$ inputs to an output of maximum $4 p$. Additionally, subtraction is implemented when sub equals 1 . The same output range is available, due to the addition of $2 p$ with use of the second RCA, after subtracting with the first RCA. Without this extra addition of $2 p$, the output would be larger than $-2 p$ and smaller than $2 p$. When $s u b=1$, all bits of $\mathrm{B}$ are inverted and the carry input of the RCA is set to 1 to obtain the 2 's complement representation of $B$. 


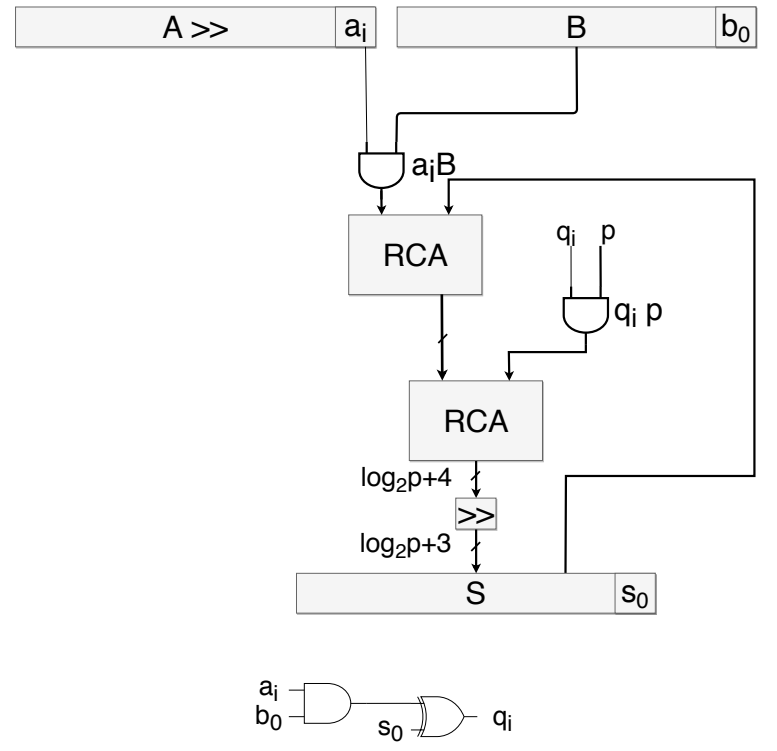

Figure 3: Architecture of MMALU when $c m d=0$.

In summary, the MMALU can perform addition and subtraction without dedicated modular addition hardware, which ensures outputs between 0 and $4 p$ when handling inputs smaller than $2 p$. Therefore, an output of the MMALU in MMM mode, which takes $4 p$ inputs and returns a $2 p$ output, can be used as input of a subsequent addition. More generally, a series of additions, subtractions and multiplications becomes possible, when the input bounds of each operation are respected. This also means that a series of subsequent additions is not always possible without intermediate scaling or MMM, which can increase the number of operations of the respective algorithm. Nevertheless, other properties of the MMM are useful for performing such a series of operations. For example, three outputs of Montgomery multiplications of values smaller than $2 p$ can be added resulting in an output smaller than $4 p$. This is because an MMM with two $2 p$ input values results in the following output bound: 


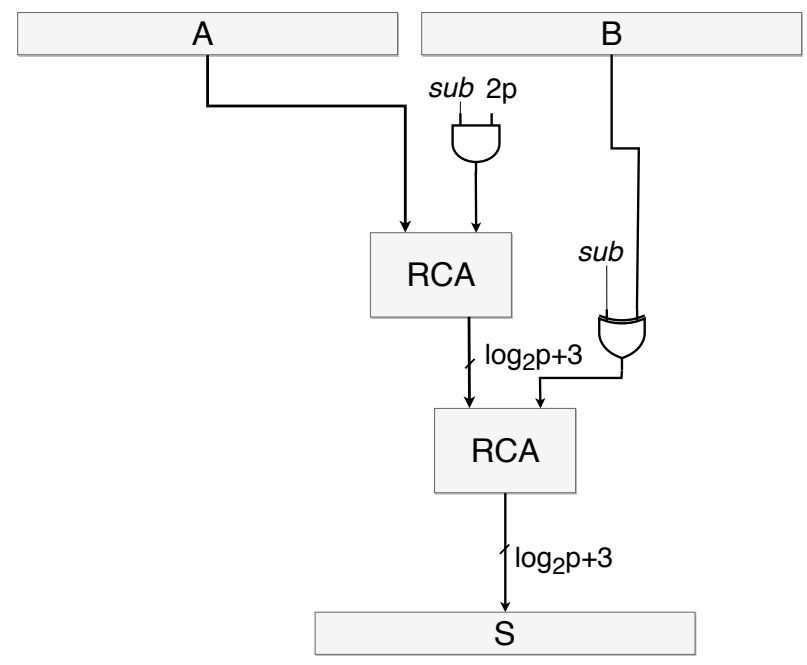

Figure 4: Architecture of MMALU when $c m d=1$.

$$
\begin{aligned}
& A, B<2 p \text { and } R>16 p \\
\stackrel{\text { Eq. }}{\Rightarrow}] & S=\frac{A B}{R}+\frac{Q p}{R} \\
\Rightarrow & S<\frac{4 \cdot p^{2}}{R}+\frac{(R-1) p}{R} \\
\Rightarrow & S<\frac{4 \cdot p^{2}}{16 \cdot p}+\frac{(R-1) p}{R} \\
\Rightarrow & S<\left(\frac{1}{4}+1\right) p-\frac{p}{R} \\
\Rightarrow & S<\frac{5}{4} p
\end{aligned}
$$

Table 3 specifies all functions of the MMALU. All calculations must respect these upper bounds on the input, otherwise a correct operation of the MMALU is not guaranteed. As a consequence of the optimizations made, not all algorithms will be directly compatible with this design, i.e. without appending scaling operations.

\subsubsection{Serialized Design of the MMALU}

When using the full-word design, the data path size and thus the implementation surface, increase with increasing key size. In 1999, Tenca and Koç 


\begin{tabular}{|l|l|l|}
\hline Functionality & Input & Output \\
\hline add/subtract & $2 \times 2 p$ & $1 \times 4 p$ \\
\hline add/subtract & $3 \times \frac{5}{4} p$ & $1 \times 4 p$ \\
\hline add & $2 \times p$ & $1 \times 2 p$ \\
\hline multiply & $2 \times 4 p$ & $1 \times 2 p$ \\
\hline multiply & $2 \times 2 p$ & $1 \times \frac{5}{4} p$ \\
\hline scale & $1 \times 4 p$ & $1 \times p$ \\
\hline
\end{tabular}

Table 3: Bounds of the inputs and outputs of the MMALU

proposed a scalable hardware architecture in [27, which can generate multiprecision results independent of the data path precision. They proposed to split the multiplicand into words in order to reduce the data path size. This resulted in the algorithm called the Multiple Word Radix-2 Montgomery Multiplication algorithm (MWR2MM), shown in Alg. 4 below. To study the influence of the scalability in Alg. 4 a second MMALU design inspired by the MWR2MM algorithm was designed.

It is important to note that the observations made in Sect. 4.1 are still applicable. Also, the MWR2MM algorithm can be parallelized by implementing pipelines, although these pipelines, while speeding up calculations, are not preferable for designs requiring minimal silicon space.

The main principle of Alg. 4 is the same as in Alg. 11, with exception of the inner loop to enable word by word scanning of the multiplicand, comprised of $e$ words of $w$ bits, i.e. $e=\left\lceil\frac{m}{w}\right\rceil$ with $m$ the number of bits of the intermediate result $S$. A subscript is used to distinguish between the different bits of a word and a superscript to indicate different words of a number. For example, $B_{k}^{(j)}$ represents the $\mathrm{k}$-th bit of the $\mathrm{j}$-th word of a number. The carry register in the inner loop needs to be at least two bits, to ensure containment during the entire iteration. This is a result of the following inequality:

$$
3\left(2^{w}-1\right)+C_{\max } \leq C_{\max } 2^{w}+2^{w}-1 \Rightarrow C_{\max } \geq 2
$$




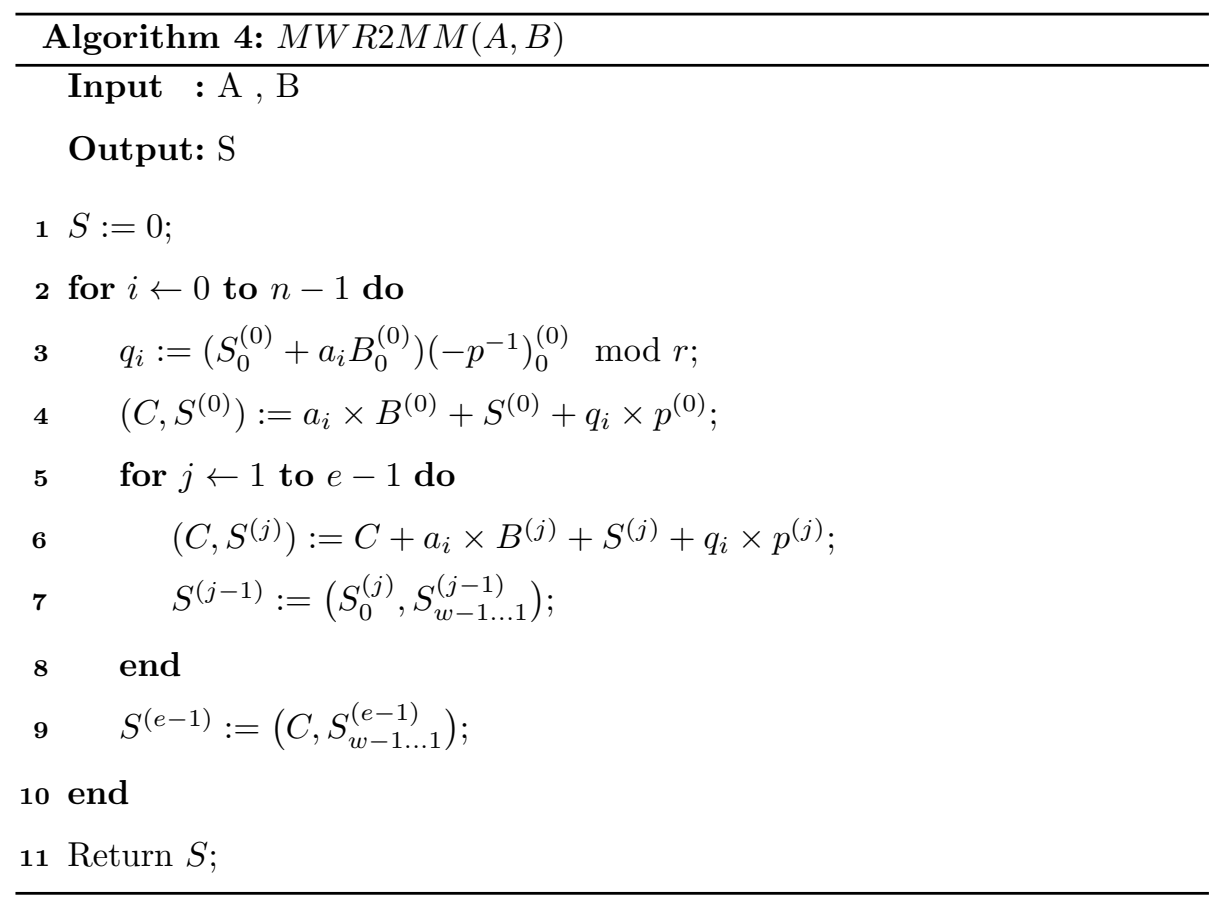

This inequality states that during the next iterations, the sum of three maximum words plus the maximum carry needs to be contained in the vector of the maximum carry concatenated with a one digit word.

The serialized MMALU is implemented with the same interface as in Fig. 2 However, carry save adders (CSA) are used to successfully pass on the carry to subsequent iterations, while scanning the inner loop. A 2-bit register is used to store the carry for the next iteration. Additionally, the adders for Montgomery multiplication are reused for addition and subtraction as was the case for the full-word design. For simplicity, a data path size of a single bit is chosen. The resulting architecture is shown in Fig. 5 for Montgomery multiplication and scaling, i.e. when $c m d=0$, and in Fig. 6 for addition and subtraction, i.e. when $c m d=1$.

Notice that for register $B$ and $S$ rotating registers were used, i.e. instead of shifting out the LSB, it becomes the MSB during the next iteration. During the innerloop in the MWR2MM algorithm, the rotating registers keep shifting, 


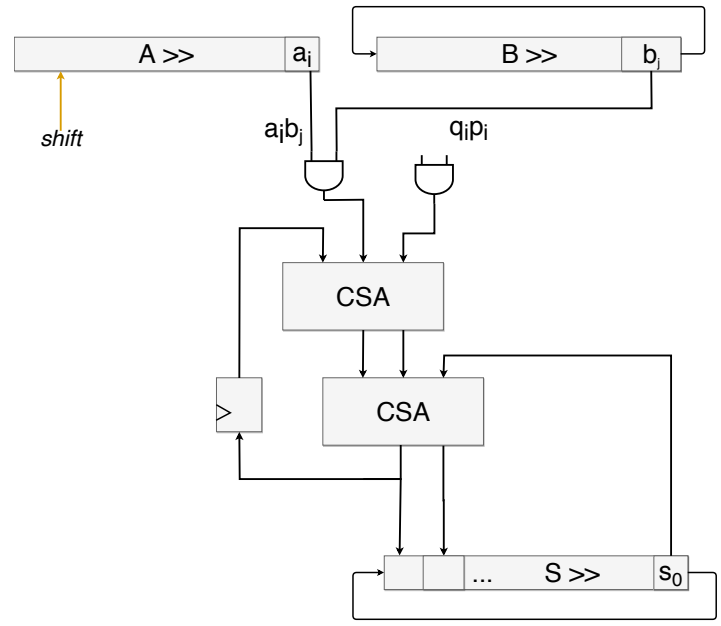

Figure 5: Architecture of the scaled MMALU when $c m d=0$

while register $A$ only shifts during the next iteration in the outerloop, i.e. when shift $=1$. As a consequence, the control logic becomes more extensive. Also, the signal $q_{i}$, which needs to remain constant while iterating over the innerloop, is stored in a flip flop and updated only on a shift pulse. Finally, it should be noted that when subtracting, the output register $S$ is initialized to $2 p$ to ensure an output in the range between 0 and $4 p$.

\subsection{Point Addition}

Moving one abstraction level up in Fig. 1, the next step is to implement the complete point addition using Algorithm 7 in 7), given in Eq. (5), using the previously designed MMALU. We use the full-word design of the MMALU for the implementation of the point addition algorithm. An overview of common architectures for ECC processor designs is given in [28. We implement a finite state machine (FSM) to implement the rules for point addition in combination with a register file to store the intermediate results. The output of the FSM consists of a write enable (WE) signal, a write address (Address) and two read adresses (LO and RO) for the register file. It also contains an OPCODE field to control the operation of the MMALU. 


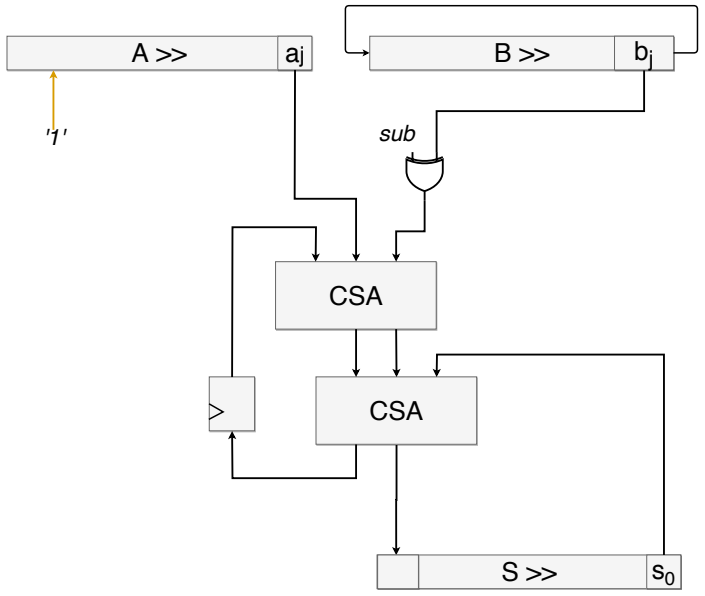

Figure 6: Architecture of the scaled MMALU when $c m d=1$

In [7], the authors already present an implementation algorithm for the proposed addition law in the form of consecutive field operations. The lifetime analysis of the intermediate values in Algorithm 7 of 7 is shown in Fig. 8(left). The figure also show the corresponding mapping of the intermediate values onto the registers in the register file. Algorithm 4 in our paper is a modified version of the sequence of operations in [7]: it takes into account the restrictions given in Table 3 and the optimizations explained in the following paragraph, resulting in a minimization of the number of registers and the size of the multiplexers.

Originally, Algorithm 7 in 7 presumes 14 registers, i.e. 6 input, 3 output and 5 temporary registers. Our design utilizes only 11 registers, reusing 3 input registers $\left(X_{1}, Y_{1}\right.$ and $\left.Z_{1}\right)$ as output registers. In addition, the algorithm ensures only 3 address bits to select the left and right operand for the MMALU and to select the write address. This is accomplished by organizing the formulas in Algorithm 4 such that maximum 8 registers need to be accessible as left or right operand. In order to reduce the number of writable registers, register $t_{0}$ is made not directly writable; instead, when writing to $t_{1}$, the value of $t_{1}$ is shifted to $t_{0}$. This is incorporated in Algorithm 4, which writes to $t_{0}$ and

\footnotetext{
${ }^{3}$ Register data of $t_{1}$ is shifted into $t_{0}\left(t_{0} \leftarrow t_{1}\right)$
} 


\begin{tabular}{|c|c|c|}
\hline \multicolumn{3}{|c|}{$\begin{array}{l}\text { Algorithm 4: Complete, projective point addition for prime order } \\
j \text {-invariant } 0 \text { short Weierstrass curves } E / F_{p}: y^{2}=x^{3}+b\end{array}$} \\
\hline \multicolumn{3}{|c|}{$\begin{array}{r}\text { Require: } P=\left(X_{1}: Y_{1}: Z_{1}\right), Q=\left(X_{2}: Y_{2}: Z_{2}\right) \\
\text { on } E: Y^{2} Z=X^{3}+b Z^{3} \text { and } b 3=3 \cdot b\end{array}$} \\
\hline \multicolumn{3}{|c|}{ Ensure: $\left(X_{3}: Y_{3}: Z_{3}\right)=P+Q$} \\
\hline 1. $t_{1} \leftarrow X_{1} \cdot X_{2}$ & 2. $t_{1} \leftarrow Y_{1} \cdot Y_{2} 3$ & 3. $t_{2} \leftarrow Z_{1} \cdot Z_{2}$ \\
\hline 4. $t_{3} \leftarrow X_{1}+Y_{1}$ & 5. $t_{4} \leftarrow X_{2}+Y_{2}$ & 6. $t_{3} \leftarrow t_{3} \cdot t_{4}$ \\
\hline 7. $t_{4} \leftarrow t_{0}+t_{1}$ & 8. $t_{3} \leftarrow t_{3}-t_{4}$ & 9. $t_{4} \leftarrow Y_{1}+Z_{1}$ \\
\hline 10. $Y_{2} \leftarrow Y_{2}+Z_{2}$ & 11. $Y_{1} \leftarrow t_{4} \cdot Y_{2}$ & 12. $Y_{2} \leftarrow t_{1}+t_{2}$ \\
\hline 13. $t_{4} \leftarrow Y_{1}-Y_{2}$ & 14. $Y_{2} \leftarrow X_{1}+Z_{1}$ & 15. $Z_{1} \leftarrow X_{2}+Z_{2}$ \\
\hline 16. $Z_{1} \leftarrow Y_{2} \cdot Z_{1}$ & 17. $Y_{1} \leftarrow t_{0}+t_{2}$ & 18. $Y_{1} \leftarrow Z_{1}-Y_{1}$ \\
\hline 19. $X_{1} \leftarrow t_{0}+t_{0}$ & 20. $t_{1} \leftarrow X_{1}+t_{0}$ & 21. $Y_{2} \leftarrow b 3 \cdot t_{2}$ \\
\hline 22. $Z_{1} \leftarrow t_{0}+Y_{2}$ & 23. $t_{1} \leftarrow t_{0}-Y_{2}$ & 24. $Y_{1} \leftarrow b 3 \cdot Y_{1}$ \\
\hline 25. $X_{1} \leftarrow t_{4} \cdot Y_{1}$ & 26. $Y_{2} \leftarrow t_{3} \cdot t_{1}$ & 27. $X_{1} \leftarrow Y_{2}-X_{1}$ \\
\hline 28. $Y_{1} \leftarrow Y_{1} \cdot t_{0}$ & 29. $Y_{2} \leftarrow t_{1} \cdot Z_{1}$ & 30. $Y_{1} \leftarrow Y_{2}+Y_{1}$ \\
\hline 31. $t_{1} \leftarrow t_{0} \cdot t_{3} \sqrt{3}$ & 32. $Z_{1} \leftarrow Z_{1} \cdot t_{4}$ & 33. $Z_{1} \leftarrow Z_{1}+t_{1}$ \\
\hline 34. $X_{1} \leftarrow \operatorname{scale}\left(X_{1}\right)$ & 35. $Y_{1} \leftarrow \operatorname{scale}\left(Y_{1}\right)$ & 36. $Z_{1} \leftarrow \operatorname{scale}\left(Z_{1}\right)$ \\
\hline
\end{tabular}

$t_{1}$ alternately. Figure 8 (right) shows the lifetime analysis and the mapping onto the register file of the intermediate values in Algorithm 4. The resulting architecture implementing the point addition is given in Fig. 7 .

\subsection{Point Multiplication}

The next design stage is implementing point multiplication with a Montgomery ladder in two variants. The first variant does not use randomization in the Montgomery ladder, as described in Alg. 2. The second variant uses randomization in the Montgomery ladder, as described in Alg. 3. Important to note is that both algorithms assume the MSB of $m$ is not 0 , therefore cutting the key space in half and making brute force attacks more feasible. This assumption 
was made to avoid addition with the point at infinity, which was not possible whilst using the typical addition formulas. However, this is not the case for their complete counterparts. Therefore, the algorithms can be altered, restoring all possibilities in the key space. In order to do so, $R_{0}$ and $R_{1}$ are initialized to $\mathcal{O}$ and $P$ respectively and the loop must iterate through all bits of $m$, i.e. from $t-1$ to 0 .

It should be noted that by using the Montgomery ladder instead of the double-and-add method, a possible speedup and reduction of resources is not feasible. The reason is that the output of a point addition is directly written in the input registers. In other words, the output of a point addition will automatically be the input for the next point addition without the need for additional loading. In the double-and-add method, one input remains unmodified. Consequently, only one input should be reloaded during the next iteration. However, the benefits of a balanced ladder method outweigh the lost speed gain and resource reduction, in spite of the lost opportunity.

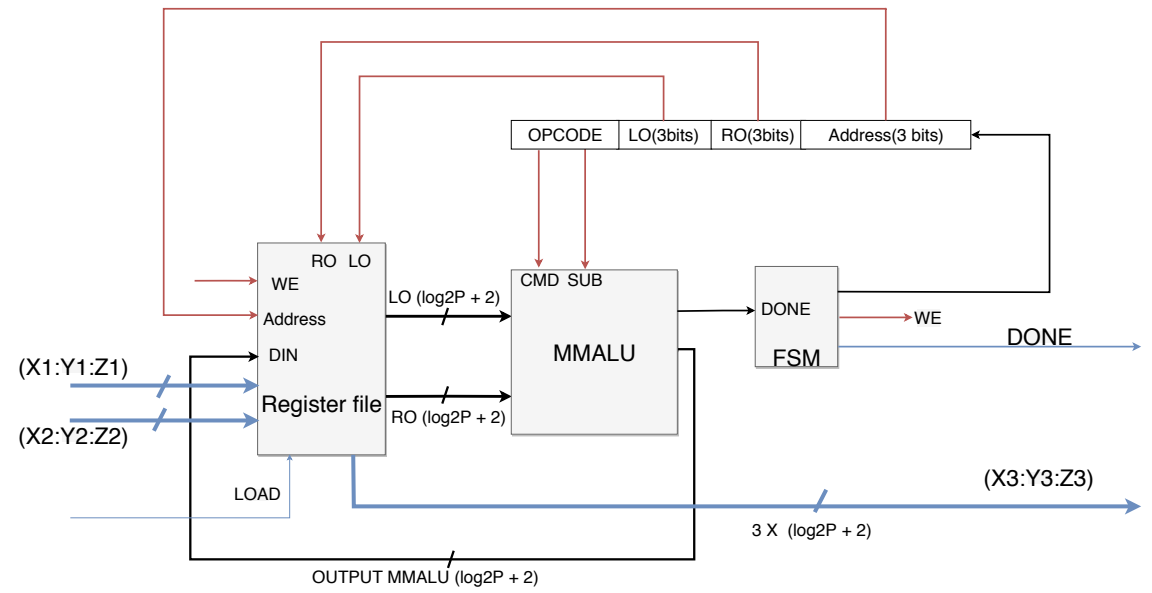

Figure 7: Architecture of the point addition module. 


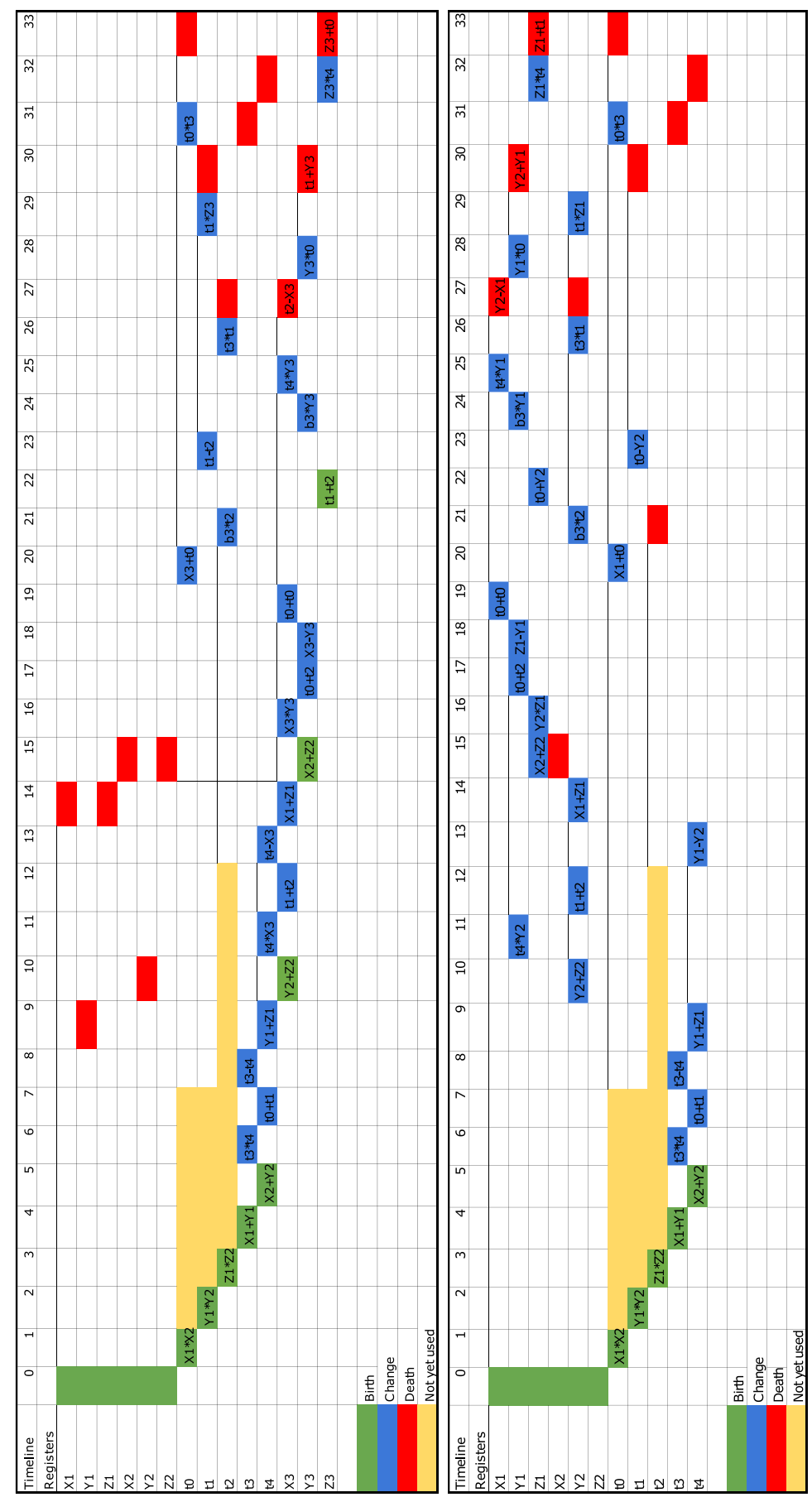

Figure 8: Lifetime analysis and mapping onto the register file of the intermediate values in Algorithm 7 in 7] (left) and in Algorithm 4 in this paper (right). 


\begin{tabular}{|l|l|l|l|l|}
\hline \# bits & $\begin{array}{l}\text { Area } \\
(\mathrm{kGE})\end{array}$ & $\begin{array}{l}\text { Area w/o regfile } \\
(\mathrm{kGE})\end{array}$ & $\begin{array}{l}\text { Max. freq. } \\
(\mathbf{M H z})\end{array}$ & $\begin{array}{l}\text { Latency } \\
(\mathbf{m s})\end{array}$ \\
\hline 64 & 17.77 & 10.03 & 333.33 & - \\
\hline 96 & 26.30 & 14.77 & 250.00 & - \\
\hline 128 & 34.12 & 18.64 & 166.67 & - \\
\hline 160 & 42.48 & 23.22 & 166.67 & $5.52(\operatorname{secp} 160 \mathrm{k} 1)$ \\
\hline 192 & 51.02 & 27.96 & 142.86 & - \\
\hline 224 & 59.12 & 32.45 & 111.11 & - \\
\hline 256 & 66.51 & 36.06 & 100.00 & $23.06(\operatorname{secp} 256 \mathrm{k} 1)$ \\
\hline
\end{tabular}

Table 4: Implementation results for the elliptic curve coprocessor based on the full-word MMALU design, without randomization in the Montgomery ladder, generated using Design Compiler 2016 with the NANGATE45 library, with and without (w/o) register file (regfile).

\section{Results}

\subsection{Implementation Results}

All blocks in the design were implemented in VHDL and simulated with ModelSim PE. The ASIC area, expressed in gate equivalents (GE), and the maximum clock frequency are calculated by Synopsis Design Compiler 2016 using the open source NANGATE45 library [29]. For testing of correctness of operation, test vectors are constructed in Magma [30. The test bench also implements the secp160k1 and secp256k1 curve used in the Bitcoin protocol.

For the implementation without randomization in the Montgomery ladder, Table 4 shows the utilized silicon area in kilo gate equivalents (kGE) and the maximum clock frequency of the design with respect to the size of the prime. For an easy comparison to future designs, the silicon area is given with and without the inclusion of the register file. Also, for the secp160k1 and the secp256k1 curves, which are described in [31, the duration of a single scalar multiplication is simulated at maximum frequency. The results show that the size of the prime and the area of the implementation are linearly correlated. Each increase of 


\begin{tabular}{|l|l|l|l|l|}
\hline \# bits & $\begin{array}{l}\text { Area } \\
(\mathrm{kGE})\end{array}$ & $\begin{array}{l}\text { Area w/o regfile } \\
(\mathrm{kGE})\end{array}$ & $\begin{array}{l}\text { Max. freq. } \\
(\mathbf{M H z})\end{array}$ & $\begin{array}{l}\text { Latency } \\
(\mathbf{m s})\end{array}$ \\
\hline 64 & 21.58 & 13.84 & 333.33 & - \\
\hline 96 & 31.91 & 20.38 & 250.00 & - \\
\hline 128 & 41.51 & 26.03 & 166.67 & - \\
\hline 160 & 51.08 & 31.81 & 166.67 & $5.52(\operatorname{secp} 160 \mathrm{k} 1)$ \\
\hline 192 & 61.35 & 38.29 & 142.86 & - \\
\hline 224 & 71.59 & 44.92 & 111.11 & - \\
\hline 256 & 81.89 & 51.45 & 100.00 & $23.06(\operatorname{secp} 256 \mathrm{k} 1)$ \\
\hline
\end{tabular}

Table 5: Implementation results for the elliptic curve coprocessor based on the full-word MMALU design, with randomization in the Montgomery ladder, generated using Design Compiler 2016 with the NANGATE45 library, with and without (w/o) register file (regfile).

32 bits for the prime, results in an increase in implementation size of approximately $6.5 \mathrm{kGE}$. In contrast, the maximum clock frequency decreases with an increasing prime size. This decline is slower than linear decrease. For completeness, Table 5 gives the results for an implementation with the randomization of the order of the point operations. Note that the speed is unaffected. However, the area increases due to the extra temporary registers in the Montgomery ladder.

Finally, Fig. 9 shows a comparison between the full-word MMALU and the serialized MMALU. The serialized MMALU with a data path size of 1 bit is significantly smaller than the full-word approach. Further, the reduction in implementation surface increases with increasing key length. However, also the duration of a single point multiplication increases. The scalable approach reaches a speed of $188.60 \mathrm{~ms}$ and $760.83 \mathrm{~ms}$ per multiplication for the secp $160 \mathrm{k} 1$ and the secp256k1, respectively. This is significantly slower than the previously obtained $5.52 \mathrm{~ms}$ and $23.06 \mathrm{~ms}$ per multiplication for the full-word MMALU. 


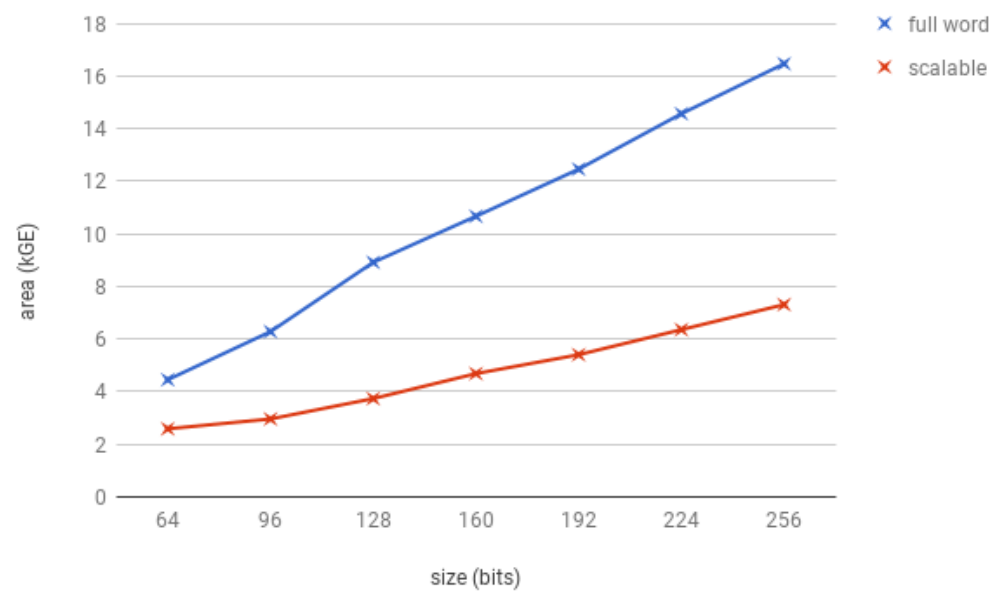

Figure 9: Comparison in silicon area between the full-word MMALU and the fully serialized MMALU.

\subsection{Side-channel resistance}

Our design is fully balanced on all design levels, from field operations to scalar multiplication, making a case for SPA resistance. Basically, different operations (such as square vs multiply in the underlying field and point add vs point double) were made indistinguishable in terms of instructions and computations performed, making their power consumption signatures identical. On top, the Montgomery ladder has some SPA resistance intrinsically built-in, thanks to the regular pattern of point operations. We strengthen this level further by randomizing the execution order and making it uncorrelated with the key bit. We expect this countermeasure to offer also some resistance against DPA attacks.

However, advanced attacks such as horizontal attacks [32] and online template attacks [33] on our implementation could still be possible. To protect against these attacks, more countermeasures are needed such as scalar and projective coordinate randomization [34, which could easily be added to our implementation without much overhead on area and latency. 


\section{Conclusions and Future Work}

This paper is the first to propose an ASIC coprocessor design for ECC with complete addition formulas in $G F(p)$ over Weierstrass curves. The design aims at SPA protection by balancing the architecture on all levels of design abstraction. We also introduce a countermeasure against DPA attacks by randomizing the execution order of the point operations in the Montgomery ladder. Our design features the following optimizations: (1) We focus on curves with $a=0$, i.e. $j$-invariant 0 curves, which lead to formulas with less computation steps. (2) We foresee two versions of the MMALU datapath with integrated adder functionality, one with a full-word width and one with serialized inputs. (3)

${ }_{425}$ We minimize the size of the register file by exploring the design parameters of the MMALU and by intelligent scheduling of the modular operations through a lifetime analysis of the intermediate variables. The silicon area, the maximum operating frequency and the scalar multiplication execution time are evaluated for the open source NANgate45 standard cell library using Synopsys Design 430 Compiler 2016.

Future work includes the practical side-channel analysis of the proposed architecture. We expect that the design will be resistant against SPA attacks and some DPA attacks. More countermeasures need to implemented, however, to provide a fully protected implementation. In addition, the latency of the presented versions of the MMALU can be further optimized by using more advanced adder units. This will lead to faster ASIC implementations of the presented coprocessor.

\section{Acknowledgements}

This work was supported in part by the Technology Foundation STW (project 44013499 - TYPHOON ASPASIA), from the Dutch government. In addition, this work was supported in part by the Central Europe Leuven Strategic Alliance (CELSA/17/033) and the Flemish government (G0E0719N). 


\section{References}

[1] P. Kocher, J. Jaffe, B. Jun, Differential power analysis, in: M. Wiener (Ed.), Advances in Cryptology - Proceedings of CRYPTO, no. 1666 in LNCS, Springer-Verlag, 1999, pp. 388-397.

[2] V. Miller, Uses of elliptic curves in cryptography, in: H. C. Williams (Ed.), Advances in Cryptology - Proceedings of CRYPTO, no. 218 in LNCS, Springer-Verlag, 1985, pp. 417-426.

[3] N. Koblitz, Elliptic curve cryptosystem, Mathematics of Computation 48 (1987) 203-209.

[4] R. L. Rivest, A. Shamir, L. M. Adleman, A method for obtaining digital signatures and public-key cryptosystems, Communications of the ACM 21 (2) (1978) 120-126.

[5] S. B. Örs, E. Oswald, B. Preneel, Power-analysis attacks on an FPGA first experimental results, in: C. D. Walter, Ç. K. Koç, C. Paar (Eds.), International Workshop on Cryptographic Hardware and Embedded Systems (CHES), no. 2779 in LNCS, Springer, 2003, pp. 35-50.

[6] D. J. Bernstein, T. Lange, Faster addition and doubling on elliptic curves, in: K. Kurosawa (Ed.), International Conference on the Theory and Application of Cryptology and Information Security (ASIACRYPT), no. 4833 in LNCS, Springer, 2007, pp. 29-50.

[7] J. Renes, C. Costello, L. Batina, Complete addition formulas for prime order elliptic curves, in: M. Fischlin, J.-S. Coron (Eds.), 35th Annual International Conference on the Theory and Applications of Cryptographic Techniques (EUROCRYPT), no. 9665 in LNCS, Springer, 2016, pp. 403428.

[8] N. Pirotte, J. Vliegen, L. Batina, N. Mentens, Design of a fully balanced ASIC coprocessor implementing complete addition formulas on Weierstrass 
elliptic curves, in: 2018 21st Euromicro Conference on Digital System Design (DSD), IEEE, 2018, pp. 545-552.

[9] P. Montgomery, Modular multiplication without trial division, Mathematics of Computation 44 (170) (1985) 519-521.

[10] C. Walter, Montgomery exponentiation needs no final subtractions, Electronics Letters 35 (21) (1999) 1831-1832.

[11] Bitcoin, https://github.com/bitcoin/bitcoin/tree/master/src/ secp256k1 (2017).

[12] P. L. Montgomery, Speeding the Pollard and elliptic curve methods of factorization, Mathematics of computation 48 (177) (1987) 243-264.

[13] L. Batina, J. Hogenboom, N. Mentens, J. Moelans, J. Vliegen, Sidechannel evaluation of FPGA implementations of binary Edwards curves, in: 17th IEEE International Conference on Electronics, Circuits, and Systems (ICECS), IEEE Publishing, 2010, pp. 1248-1251.

[14] L. Batina, N. Mentens, B. Preneel, I. Verbauwhede, Balanced point operations for side-channel protection of elliptic curve cryptography, IEE Proceedings-Information Security 152 (1) (2005) 57-65.

[15] D. J. Bernstein, P. Birkner, M. Joye, T. Lange, C. Peters, Twisted Edwards curves, in: S. Vaudenay (Ed.), International Conference on Cryptology in Africa (AFRICACRYPT), no. 5023 in LNCS, Springer, 2008, pp. 389-405.

[16] D. J. Bernstein, C. Chuengsatiansup, D. Kohel, T. Lange, Twisted Hessian curves, in: K. Lauter, F. Rodríguez-Henríquez (Eds.), International Conference on Cryptology and Information Security in Latin America (LATINCRYPT), no. 9230 in LNCS, Springer, 2015, pp. 269-294.

[17] P. M. C. Massolino, J. Renes, L. Batina, Implementing complete formulas on Weierstrass curves in hardware, in: C. Carlet, M. A. Hasan, V. Saraswat 
(Eds.), International Conference on Security, Privacy, and Applied Cryptography Engineering (SPACE), no. 10076 in LNCS, Springer, 2016, pp. 89-108.

[18] Ł. Chmielewski, P. M. C. Massolino, J. Vliegen, L. Batina, N. Mentens, Completing the complete ECC formulae with countermeasures, Journal of Low Power Electronics and Applications 7 (1).

[19] A. P. Fournaris, C. Dimopoulos, A. Moschos, O. Koufopavlou, Design and leakage assessment of side channel attack resistant binary edwards elliptic curve digital signature algorithm architectures, Microprocessors and Microsystems 64 (2019) 73-87.

[20] L. Batina, Arithmetic and architectures for secure hardware implementations of public-key cryptography, $\mathrm{PhD}$ thesis (2005).

[21] S. B. Örs, L. Batina, B. Preneel, J. Vandewalle, Hardware implementation of a Montgomery modular multiplier in a systolic array, in: International Parallel and Distributed Processing Symposium, IEEE, 2003.

[22] L. Batina, N. Mentens, S. B. Ors, B. Preneel, Serial multiplier architectures over $\operatorname{GF}\left(2^{n}\right)$ for elliptic curve cryptosystems, in: Electrotechnical Conference, 2004. MELECON 2004. Proceedings of the 12th IEEE Mediterranean, Vol. 2, IEEE, 2004, pp. 779-782.

[23] N. Mentens, K. Sakiyama, B. Preneel, I. Verbauwhede, Efficient pipelining for modular multiplication architectures in prime fields, in: 17th ACM Great Lakes symposium on VLSI, ACM, 2007, pp. 534-539.

[24] F. Bernard, Scalable hardware implementing high-radix Montgomery multiplication algorithm, Journal of Systems Architecture 53 (2) (2007) 117126.

[25] K. Sakiyama, N. Mentens, L. Batina, B. Preneel, I. Verbauwhede, Reconfigurable modular arithmetic logic unit supporting high-performance RSA 
and ECC over GF(p), International Journal of Electronics 94 (5) (2007) 501-514.

[26] K. Sakiyama, L. Batina, N. Mentens, B. Preneel, I. Verbauwhede, Smallfootprint ALU for public-key processors for pervasive security, in: Workshop on RFID Security, Vol. 12, 2006.

[27] A. Tenca, C. Koc, A scalable architecture for Montgomery multiplication, Cryptographic Hardware And Embedded Systems 1717 (1999) 94-108.

[28] H. Marzouqi, M. Al-Qutayri, K. Salah, Review of elliptic curve cryptography processor designs, Microprocessors and Microsystems 39 (2) (2015) $97-112$.

[29] NanGate Inc, NanGate FreePDK45 Open Cell Library (2018).

[30] Magma computational algebra system (2018). URL http://magma.maths.usyd.edu.au/magma/

[31] Certicom Corp., SEC 2: Recommended elliptic curve domain parameters (2000).

URL http://www.secg.org/SEC2-Ver-1.0.pdf

[32] A. Bauer, E. Jaulmes, E. Prouff, J. Wild, Horizontal and vertical sidechannel attacks against secure rsa implementations, in: E. Dawson (Ed.), Topics in Cryptology - CT-RSA 2013, Springer Berlin Heidelberg, Berlin, Heidelberg, 2013, pp. 1-17.

[33] L. Batina, Ł. Chmielewski, L. Papachristodoulou, P. Schwabe, M. Tunstall, Online template attacks, Journal of Cryptographic Engineering 9 (1) (2019) $21-36$.

[34] J. Coron, Resistance against Differential Power Analysis for Elliptic Curve Cryptosystems, in: Cryptographic Hardware and Embedded Systems, First International Workshop, CHES'99, Worcester, MA, USA, August 12-13, 1999, Proceedings, 1999, pp. 292-302. 